第 622 次学术讨论会 - 未来地球计划与人类命运共同体建设

\section{全球能源互联网及关键技术}

\section{周原冰}

全球能源互联网发展合作组织, 北京 100031

E-mail: zhouyuanbing@geidco.org

以全球变暖为主要特征的气候变化对自然生态系统 和人类社会产生巨大影响, 正日益威胁人类可持续发展. 工业革命以来以化石能源为主导的能源供应体系是温室 气体排放的主要来源, 且随着全球能源需求的快速增长, 全球温室气体排放也呈现爆发式增长. 当前能源发展方式 的不可持续性, 主要体现在资源、环境和气候三个方面. 首先是资源不可持续, 按照目前开采强度, 煤油气资源仅 能继续开采 50 100 年; 其次是环境不可持续, 化石能源的 大量开发使用, 对大气、土壤、地下水等造成污染, 对地 质造成破坏; 最后是气候不可持续, 全球气温升高、海平 面上升, 已经成为人类社会生存和可持续发展的重大威 胁 ${ }^{[1]}$.

全球气候变暖主要源于温室气体排放导致的温室效 应不断累积, 主要有二氧化碳、氧化亚氮、甲烷等. 其中 化石能源燃烧排放的二氧化碳是形成温室效应的主要原 因. 截至 2016 年, 人类活动已导致大气中二氧化碳浓度上 升至 $403 \mathrm{ppm}$ 左右 $\left(1 \mathrm{ppm}=1000 \mathrm{mg} / \mathrm{m}^{3}\right.$ )(图 1), 刷新了近 300 万年来的纪录, 较工业革命前水平升高了 $45 \%^{[2]}$.

二氧化碳是温室气体的主要成分. 按照二氧化碳当量 计算, 2014 年二氧化碳占总温室气体排放量的 $77 \%$, 主要 来源于化石燃料的燃烧, 包括电力、交通、建筑、工业和 农业等行业对化石燃料的消耗. 与化石能源利用相关的二 氧化碳排放约占二氧化碳总排放的 $85 \%^{[3,4]}$, 对全球变暖 具有决定性作用(图 2).

实现世界范围内的能源低碳、零碳是应对气候变化的 根本出路. 当前全球超过 $80 \%$ 的能源消费为化石能源, 如 果不改变现有能源发展方式, 预计 21 世纪末全球能源累 计碳排放将超过 4.8 万亿吨, 温升有可能超过 $3^{\circ} \mathrm{C}$. 而构建 全球能源互联网, 正是实现能源清洁转型、可持续发展的 根本途径, 能够为实现《巴黎协定》提出的 $2^{\circ} \mathrm{C}$ 温控长期 目标提供全球减排路径, 以电网互联互通加速全球清洁能 源高效、规模化开发利用, 预计 21 世纪可将全球碳排放从 约 4.8 万亿吨有效控制到 1 万亿吨左右, 实现 $2^{\circ} \mathrm{C}$ 温控目标 (图 3).

本文基于全球能源现状分析，提出应对能源挑战的世 界能源转型思路, 介绍了全球能源互联网理念与构建设想, 提出了全球能源互联网面临的关键问题.

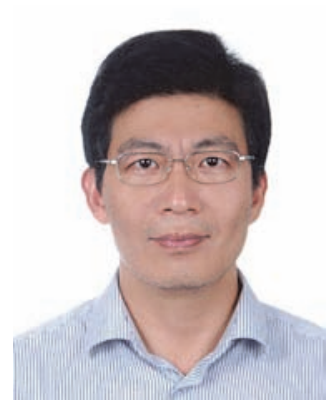

周原冰现任全球能源互联 网发展合作组织经济技术研究院 院长。享受国务院特殊津贴专家。 中国可再生能源学会理事、可再 生能源发电并网专业委员会副主 任委员, 央企智库联盟专家委员 会委员。长期从事能源电力发展 战略规划、能源经济政策、企业 战略管理、新能源与智能电网、 全球能源互联网等研究工作。主持和参与能源电力发展规 划研究、国际化发展、新能源与电网协调发展、分布式能 源、体制改革与电力市场研究等重大课题近百项.

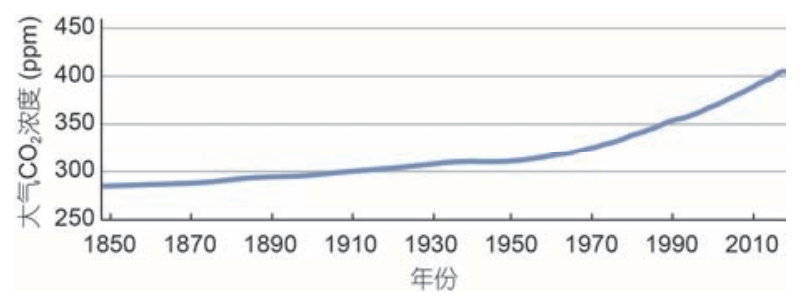

图 1 1850 2017 年全球大气二氧化碳平均浓度变化(ppm)

Figure 1 Average concentration change in atmospheric carbon dioxide from 1850 to 2017

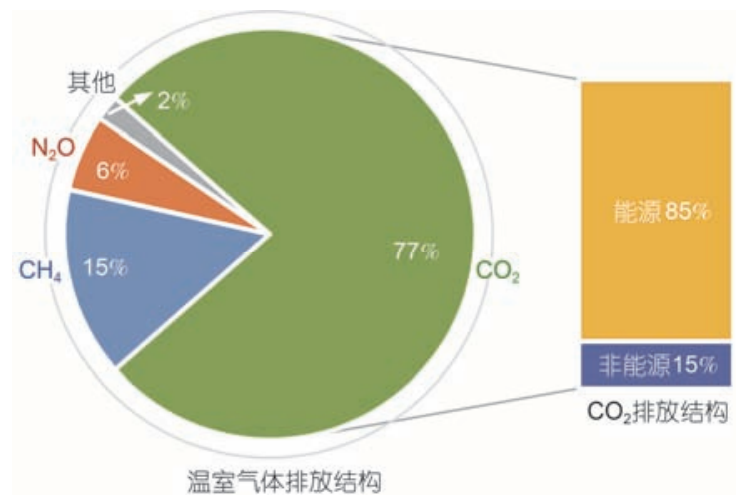

图 22014 年全球温室气体排放及 $\mathrm{CO}_{2}$ 排放结构

Figure 2 Composition of global emissions of greenhouse gases and $\mathrm{CO}_{2}$ in 2014 


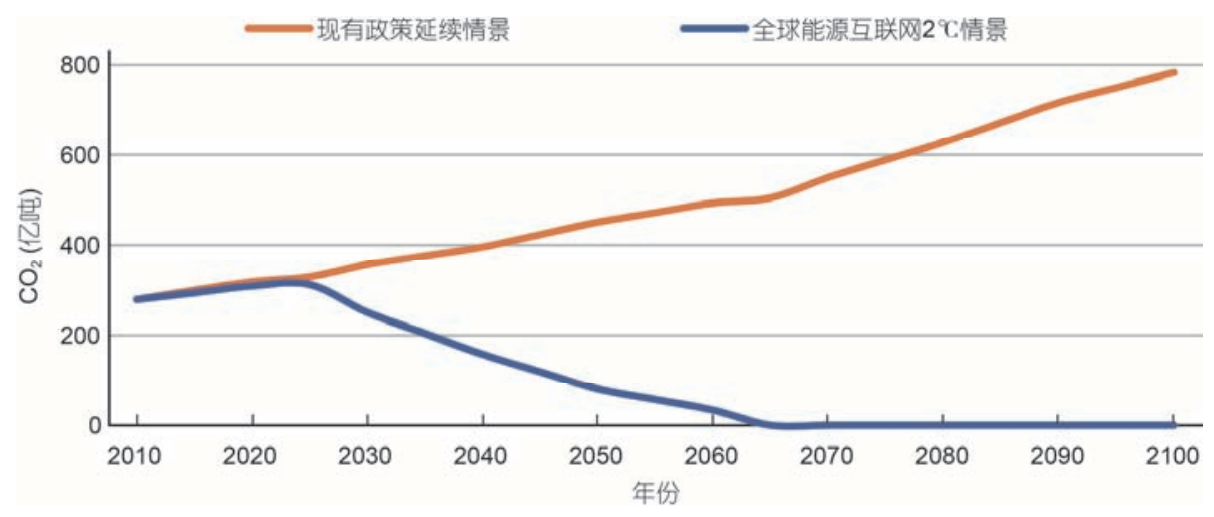

图 3 现有政策延续情景与全球能源互联网 $2^{\circ} \mathrm{C}$ 情景对应的全球能源系统排放路径

Figure 3 Global $\mathrm{CO}_{2}$ emission path of energy sector in $\mathrm{BAU}$ scenario and $\mathrm{GEI} 2^{\circ} \mathrm{C}$ scenario

\section{1 全球能源互联网}

全球能源互联网 ${ }^{[5]}$ 是推动能源可持续发展, 实现以清 洁化、电气化、网络化为特征的能源转型, 加快实施“两个 替代、一个提高、一个回归”, 构建清洁主导、电为中心、 互联互通、共建共享的全球能源互联网, 能够保障人人享 有清洁、可靠、可负担的现代能源供应.

全球能源互联网是清洁能源在全球范围大规模开发、 输送、使用的重要平台, 是加快能源转型和电力发展方式 转变, 实现世界能源清洁低碳、安全高效和可持续发展的 根本解决方案. 构建全球能源互联网, 一方面可以构筑能源 发展新格局, 突破资源、时空和环境约束, 实现人人享有充 足清洁能源, 高效利用清洁能源, 清洁能源主导人类能源 供给; 另一方面可以激发经济增长新活力, 增强发展动 力、释放创新红利、推动合作共赢, 促进世界经济协调发 展; 同时还可以创造全球社会美好新生活, 改善自然环境, 实现生态可持续发展, 推动人类和谐发展, 促进文明升级.

全球能源互联网实质就是智能电网+特高压电网+清洁 能源. 清洁能源是根本, 全球主要流域水能、北极风能和 赤道太阳能以及各国集中式和分布式清洁能源将成为主导 能源, 是实现绿色低碳发展的资源保证; 特高压电网是关键, 能够实现数千公里、万兆瓦级电力输送和跨洲、跨区、跨国 电网互联, 全球各大清洁能源基地和用电地区都在特高压 电网覆盖范围内; 智能电网是基础, 能够适应各类集中 式、分布式清洁能源的灵活接人, 实现源网荷储协同优化、 多能互补和高效使用, 满足用户多样化需求.

世界范围内, 在新一轮能源革命的驱动下, 各主要国 家都在积极发展智能电网和清洁能源, 推进电网互联, 成 为构建全球能源互联网的重要基础. 20 世纪 60 年代起, 苏 联、日本、美国、意大利等国家先后提出发展特高压输电 技术, 开展了相关规划、设计、试验和设备研制等工作, 取 得了一定成果. 进人 21 世纪, 各国愈发重视智能电网的发 展, 围绕电网网架、高级量测系统、电动汽车基础设备和储
能技术等 4 个重点领域展开研究，期望通过智能电网有效 应对能源、环境气候等方面的挑战, 保证安全、可靠、优 质、高效的电力供应, 满足互动、多样的电力需求. 21 世 纪以来, 中国经济持续快速发展, 能源需求不断增长, 推 动大型煤电、水电、核电以及风电、太阳能发电为主的可 再生能源基地加快建设. 同时, 由于中国能源资源和能源 消费呈现地域逆向分布的特点, 迫切需要大规模、大范围 优化配置资源, 发挥坚强智能电网大容量、远距离输电优 势, 实现各类能源基地的集约开发和外送消纳. 近年来, 中国大力推动特高压、智能电网、清洁能源的发展, 在坚 强智能电网的技术创新、标准制定、战略规划、工程建设 等方面开展了大量工作, 为全球能源互联网发展储备了技 术、创造了条件.

\section{2 总体设想及建设规划}

\section{1 总体设想}

全球能源互联网以特/超高压输电、柔性直流输电和海 底电缆等先进技术为支撑, 由跨洲、跨国骨干网架和各国 各电压等级电网构成, 连接各大洲大型能源基地, 适应各 种集中式、分布式电源, 能够将风能、太阳能、海洋能等 可再生能源输送到各类用户, 是服务范围广、配置能力强、 安全可靠性高、绿色低碳的全球能源配置平台, 具有网架 坚强、广泛互联、高度智能、开放互动等特征. 各大洲能 源互联网是构建全球能源互联网骨干网架的基础. 总体上, 未来全球能源互联网发展可以划分为洲内互联、跨洲互联 和全球互联 3 个发展阶段 ${ }^{[6]}$. 目前至 2025 年, 推动形成全 球能源互联共识; 2025 2035 年, 根据技术经济比较优势, 启动大型清洁能源基地建设, 加强洲内国家之间的电网互 联; 2035 2050 年, 在继续加强各洲主要国家电网互联的基 础上, 按照先易后难顺序, 推动大型能源基地开发和跨洲 联网取得重要进展; 2050 2070 年, 加快全球能源互联网建 设, 逐步形成全球互联格局，推动实现“两个替代”目标. 
结合各国各洲当前能源和电网发展现状, 其未来发展 重点如下: (1) 亚洲的发展重点是加快东南亚、南亚电网建 设, 提高电力普及性, 解决经济发展的电力需求和无电人 口问题; 加快东南亚水电、西亚太阳能等大型清洁能源基 地开发, 将资源优势转化为经济优势; 中国发挥特高压技 术优势, 构建国内能源互联网. (2) 欧洲的发展重点是坚持 清洁能源开发与洲外输电并重, 加强输电通道建设, 提升 电网传输效率, 支撑清洁能源大规模开发利用, 推进亚欧 非联网, 扩大能源供给. (3) 非洲的发展重点是满足自身电 力需求, 加强能源电力基础设施建设, 解决经济发展的电力 需求和无电人口问题; 通过加快水电等清洁能源开发, 将资 源优势转化为经济优势, 促进洲内及跨洲联网. (4) 美洲的 发展重点是加快电网升级改造、加强电网互联, 实现清洁能 源大规模开发和大范围配置, 提升电网安全可靠水平.

基于资源禀赋、能源电力需求和气候环境治理需要, 通 过在各国骨干网架和跨国联网基础上进一步加强洲际联网, 未来全球将形成“九横九纵”能源互联网骨干网架，包括亚 欧非“四横六纵”互联通道、美洲“四横三纵”互联通道和北极 能源互联通道, 将广泛互联大型清洁能源基地与负荷中心, 实现清洁能源全球配置, 跨时区、跨季节大规模互济.

\section{2 建设规划}

全球能源互联网骨干网架的建设总体分为 2035 、2050
和 2070 三个规划阶段 ${ }^{[6 ~ 9]}$.

预计到 2035 年建成“五横五纵”互联通道(图 4), 亚洲欧洲-非洲率先实现跨洲联网. 初步建成亚欧、亚欧非和美 洲骨干网架，新增输电线路 $68000 \mathrm{~km}$ 、输电容量 $280 \mathrm{GW}$, 其中跨海输电长度为 $6660 \mathrm{~km}$ 、跨海输电总容量为 $91.5 \mathrm{GW}$.

预计到 2050 年建成“七横七纵”互联通道(图 5), 基本建 成全球能源互联网骨干网架, 形成清洁能源全球开发、配置 和使用新格局. 完善亚欧、亚欧非、美洲骨干网架，同时建 设北极能源互联网骨干网架, 合计新增输电线路 109000 $\mathrm{km}$ 、输电容量 $435 \mathrm{GW}$, 其中跨海输电长度为 $4190 \mathrm{~km}$ 、跨 海输电总容量为 $112 \mathrm{GW}$.

预计到 2070 年，在 2050 年“七横七纵”互联通道的基 础上, 进一步加强洲际联网, 全球建成“九横九纵”全球能 源互联网骨干网架(图 6), 广泛互联大型清洁能源基地与 负荷中心, 实现清洁能源全球配置, 跨时区、跨季节大规 模互济。“九横九纵”骨干网架包括亚欧非“四横六纵”互联 通道、美洲“四横三纵”互联通道和北极能源互联通道，总 输电长度超过 $180000 \mathrm{~km}$, 覆盖全球 100 多个国家、超过 $80 \%$ 世界总人口和 $90 \%$ 经济总量 ${ }^{[7]}$.

\section{3 技术基础与综合效益}

构建全球能源互联网, 核心技术基础包括大规模清 洁能源开发技术、特高压输电技术和智能电网技术三方

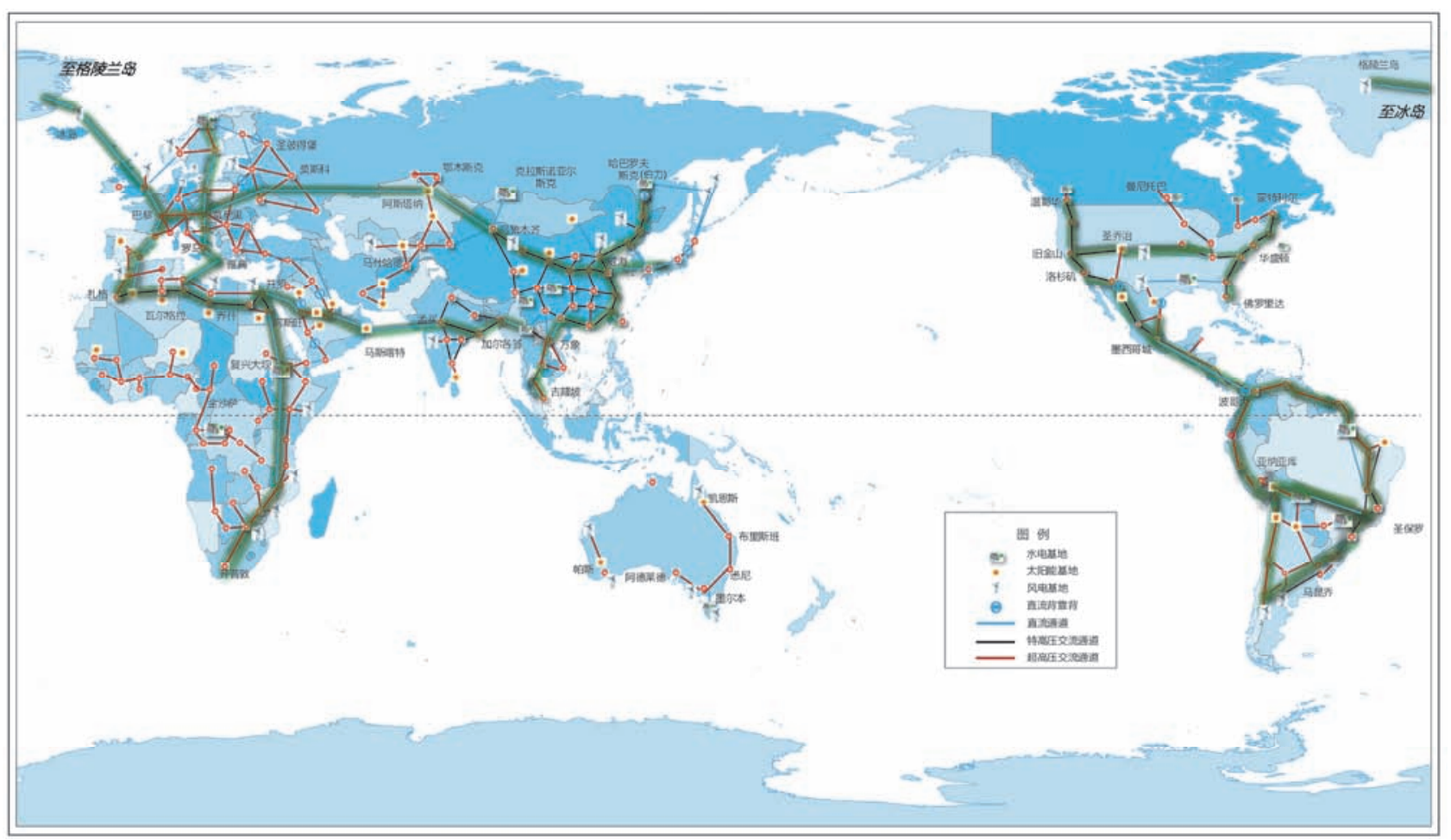

图 42035 年“五横五纵”互联通道

Figure 4 Schematic diagram of " 5 Horizontal and 5 Vertical" Global Energy Interconnection backbone grid in 2035 


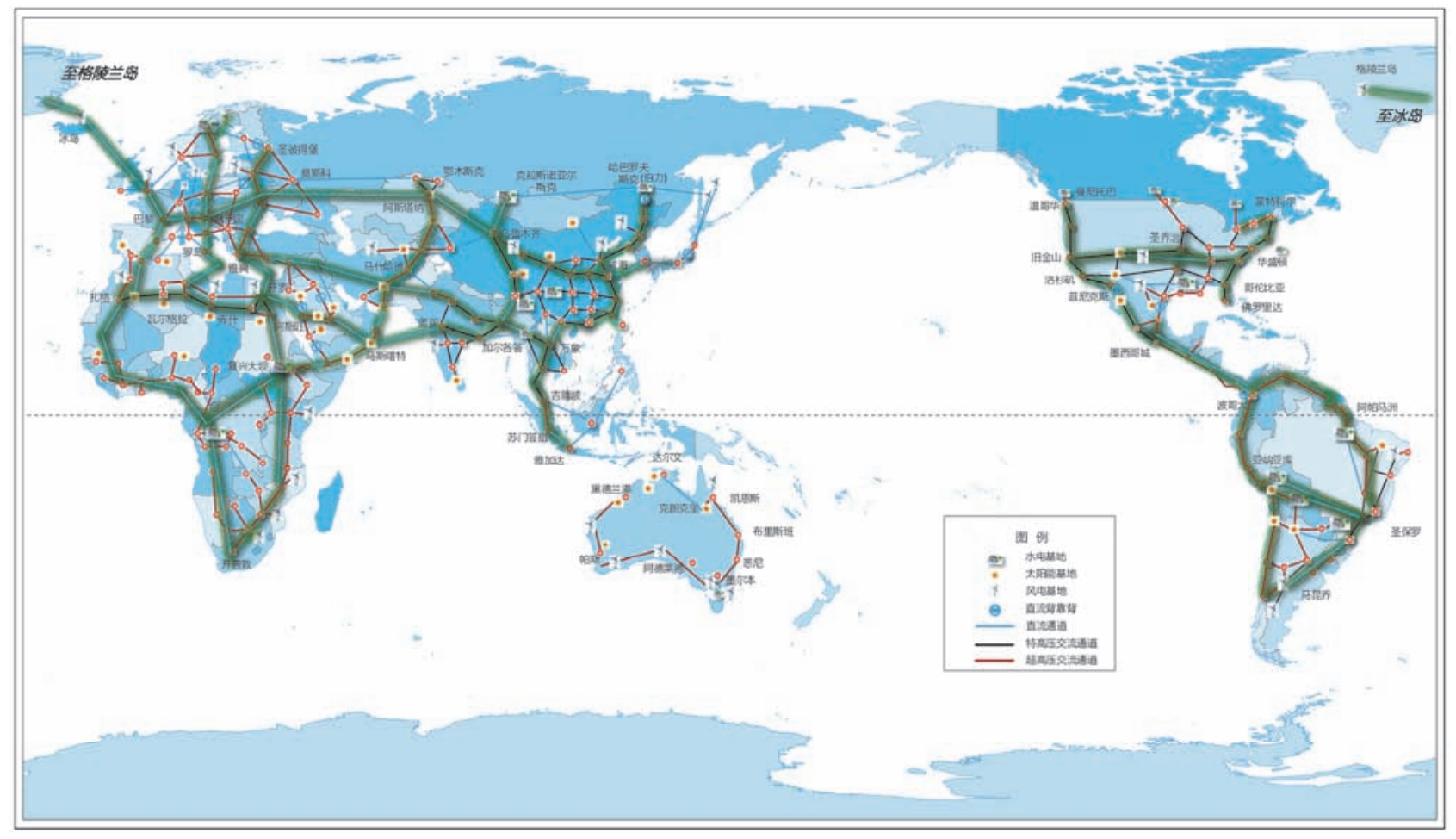

图 52050 年“七横七纵”互联通道

Figure 5 Schematic diagram of "7 Horizontal and 7 Vertical" Global Energy Interconnection backbone grid in 2050

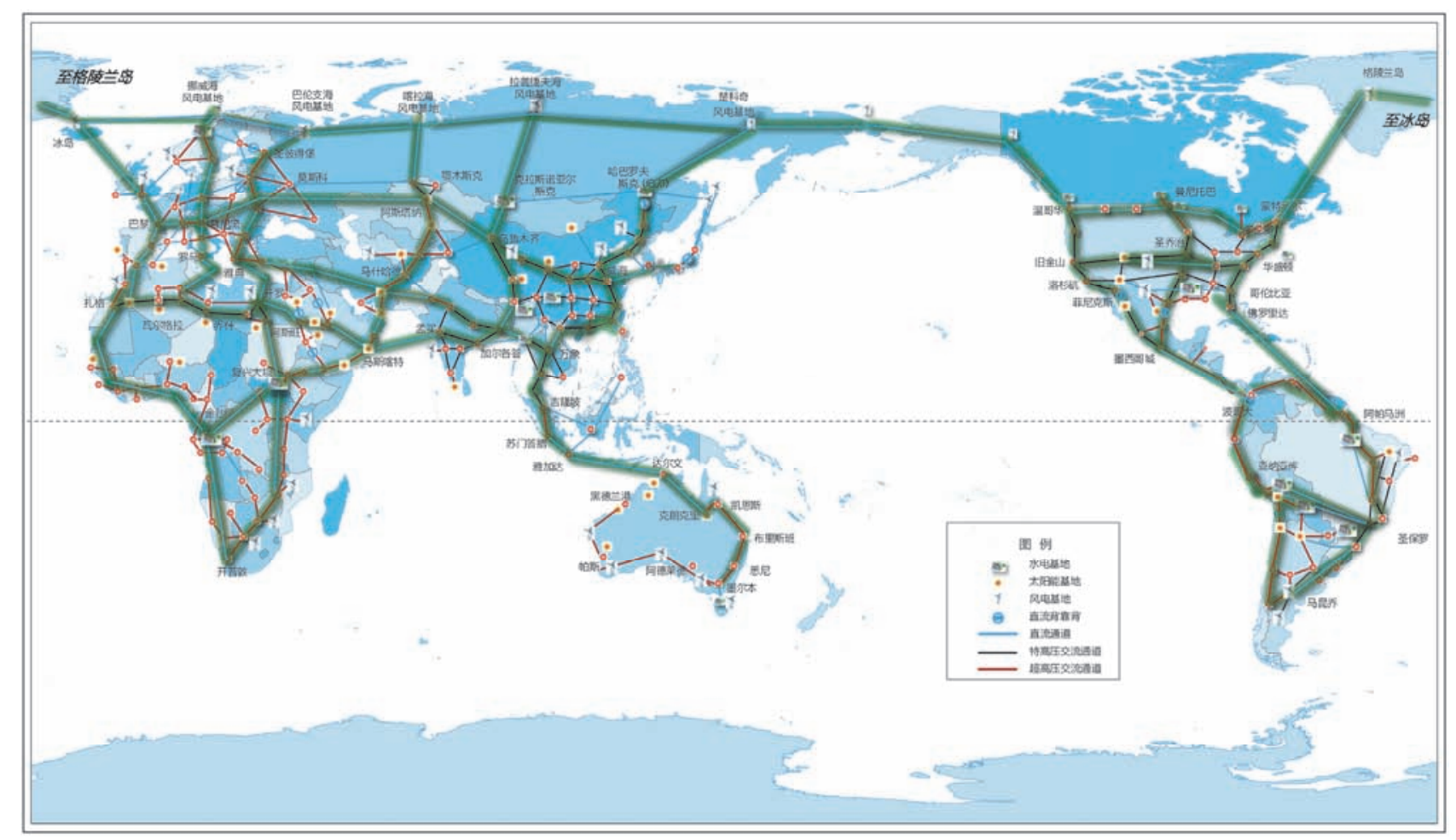

图 62070 年“九横九纵”互联通道

Figure 6 Schematic diagram of "9 Horizontal and 9 Vertical" Global Energy Interconnection backbone grid in 2070 
面. 全球清洁能源具有资源丰富、分布不均的特点, 资源 富集地区大都远离用电负荷地区, 需要就地转化为电能、 远距离输电, 同时风电、光伏发电具有间歇性、波动性, 需 要融人大电网才能实现大发展.

构建全球能源互联网, 是实现清洁能源高效开发、配 置和利用的有效解决方案, 其核心任务是建设清洁能源大 规模开发、全球配置、互补互济、高效利用的战略通道 ${ }^{[8]}$. 应进一步完善清洁能源开发技术, 在充分开发基础上提高 利用效率、提高并网友好性、降低对大电网的扰动和冲击. 近年来, 特高压技术和智能电网技术快速发展, 对最先进 的 $\pm 1100 \mathrm{kV}$ 直流输电技术而言, 其输电距离可达 6000 $\mathrm{km}$ 、容量可达 $15 \mathrm{GW}$. 随着电网智能控制、大规模储能等 技术的不断突破, 电网能够适应清洁能源大规模接人并保 障安全运行, 因而构建全球能源互联网已具备技术可行性. 全球能源互联网的构建, 要满足各种形态可再生能源灵活 接人的需求, 能够充分利用不同能源资源时空互补性, 将 全世界范围的清洁能源生产和消费市场联系起来. 依托全 球能源互联网骨干网架, 加快清洁能源开发利用, 对满足 全球电力需求、拉动世界经济增长、降低全球电价水平、 应对气候变化、保护和改善生态环境、创造社会美好生活 以及支撑服务人类命运共同体建设等具有重要意义.

根据全球能源互联网发展合作组织 2018 年发布的全 球骨干网架规划研究成果, 全球能源互联网构建具有极高 的综合效益, 预计累计总投资约 38 万亿美元, 拉动全球经 济增长率年均可达 0.2 个百分点. 对非洲经济的拉动作用 最为明显, 其经济增长率年均可达 0.4 个百分点. 2050 年, 全球平均度电成本将比目前降低约 2.8 美分/千瓦时, 每年 节约电费约 1.8 万亿美元. 其中, 欧洲和非洲平均度电成 本降幅最大, 分别可降低 3.8 美分/千瓦时和 3.2 美分/千瓦 时. 2018 年 2050 年, 能源二氧化碳累计排放可控制在 8000 亿吨左右. 2070 年前, 预期将实现能源二氧化碳净零 排放. 总体上, 每年可减少二氧化硫排放 1.2 亿吨、氮氧化 物排放 1.9 亿吨、细颗粒物排放 3900 万吨, 节约发电用水 740 亿 $\mathrm{m}^{3}$ 以上, 大幅降低化石能源的开发利用规模, 使开 采、加工、运输、存储、燃烧等过程中引起的地下水污染、 地质破坏、陆地和海洋生态破坏日益减轻, 生态环境将得 到保护和恢复 ${ }^{[9]}$.

\section{3 关键技术研究}

全球能源互联网建设关键技术主要包括基于多能互 补的清洁能源发电及储能、特高压柔性直流输电、高温超 导输电及交直流混联大电网运行控制等技术及其对应核 心设备技术 ${ }^{[10]}$.

\section{1 基于多能互补的清洁能源发电及储能技术}

清洁能源具有波动性、不稳定性和低惯性等特点, 其
未来发展将以多能互补为主要特征. 近年来清洁能源的规 模开发及并网得到了快速发展, 但其在当前电网中的整体 占比仍相对较小, 面临着诸多送出、消纳问题. 随着清洁 能源发电基地电力大规模外送和分布式清洁电源渗透率 不断增加, 未来电网将更大规模、更大范围并以更高比例 地接收和消纳清洁能源, 需要开展高比例清洁能源发电并 网、大规模储能及多能互补协调运行控制等方面研究, 典 型技术包括清洁能源功率预测、大规模储能和虚拟同步机 技术等.

\subsection{1 清洁能源功率预测}

清洁能源功率预测主要基于历史功率数据、实时气 象、数值天气预报、地形地貌和设备状态等数据建立功率 预测模型, 并根据应用需求不同, 给出不同时间尺度 (超短 期、短期和中长期)的预测功率值. 多年来国内外各研究机 构相继提出了物理预测、统计预测和混合预测等多种功率 预测技术, 并常采用直接测量、数值模拟与卫星反演等方 法进行风能、太阳能资源评估.

总体上，清洁能源功率预测技术整体上发展较为迅速， 但针对全球能源互联网情景下不同大洲及区域的资源分 布特性差异、发电特性差异及其影响下的电能输送与并网 控制等难题, 仍需要解决清洁能源资源评估及功率预测的 全球覆盖问题，并进一步提升对于大规模清洁能源基地的 预测能力和精度水平.

\subsection{2 大规模储能技术}

大规模间歇性可再生能源发电的接入使得电源侧不 确定性增加, 增大电网功率不平衡引发的风险. 高比例清 洁能源并网情景下, 一方面可通过储能与清洁能源发电联 合运行, 减少随机性并提高可控性; 另一方面通过电网级 的储能应用, 增强电网对清洁能源发电的适应性, 此时储 能作为电网的可调度资源, 将具有更大的应用价值和应用 空间. 典型储热系统示意图如图 7 所示.

电网级应用中的储能需求可分为功率服务和能量服务 两类：(1) 功率服务中, 储能用于应对电网的暂态稳定及短 时功率平衡需求, 作用时间从数秒至数分钟, 技术上要求快 速响应的大容量存储, 如电池储能、飞轮储能、超级电容储 能等. (2) 能量服务中, 储能用于长时间尺度的功率调节, 作 用时间从数小时延伸至季节时间尺度, 用于应对系统峰谷调 节以及输配电线路的阻塞问题, 技术上要求长时间尺度存 储、较高循环效率及较低成本, 以实现可再生能源发电在时 间维度上的转移, 如抽水蓄能、压缩空气等. 储氢、储热等 单向大规模储能技术可有效利用冗余新能源发电, 并在长时 间尺度上为广域能源互联网运行提供支持.

目前, 以蓄电池、飞轮、压缩空气和抽水蓄能为代表 的电能大规模存储设备高速发展, 但仍受限于存储容量尚 无法满足并改善超大规模清洁能源的间歇式接人性能, 特 别是具有快响应速度的蓄电池储能, 储能容量亟待提高, 需要进一步研究开发. 


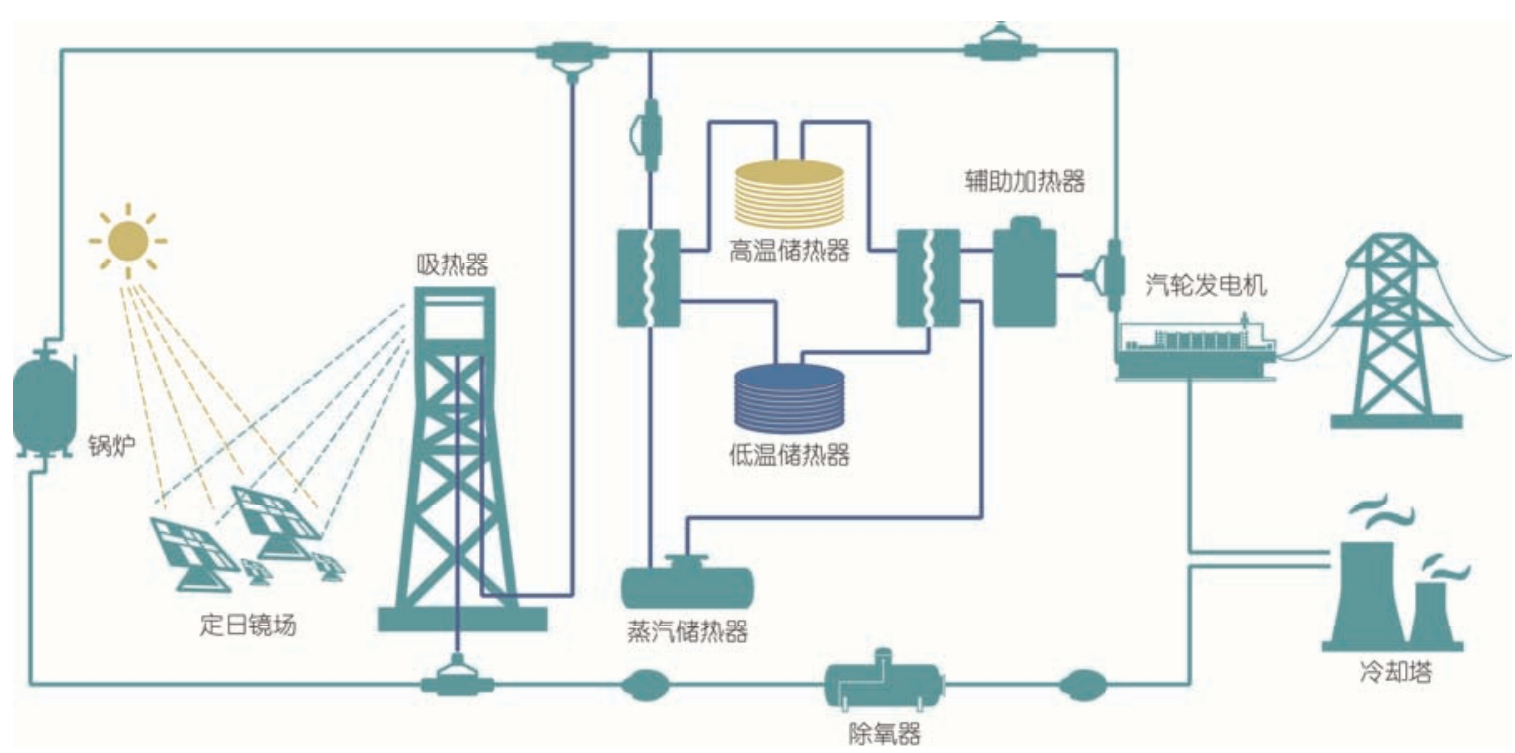

图 7 储热系统示意图

Figure 7 Schematic diagram of a thermal storage system

\subsection{3 虚拟同步机技术}

虚拟同步发电机技术通常指含有电力电子变流器的 电源的控制环节, 采用同步发电机组的机电暂态转子运动 方程, 使采用该技术的装置并网运行时具有同步发电机组 并网运行的一次调频特性、无功调压特性、惯性、阻尼特 性等运行外特性的技术.

清洁能源虚拟同步机分为分散式与集中式两类，可提 高逆变器的并网友好性、改善新能源并网稳定性, 是抑制 光伏多机并网谐振等问题的核心技术. 分散式包括光伏虚
拟同步机与风机虚拟同步机(图 8), 单机分散接人电网使每 个发电单元具备同步发电机特性; 集中式虚拟同步机在电 站并网点接人, 使新能源电站在电网运行控制响应上呈现 与同步机相近的外特性. 中国张北国家风光储输示范工程 是全球容量最大的虚拟同步机示范工程，风机虚拟同步机 $436 \mathrm{WM}$ 、光伏虚拟同步机 $12 \mathrm{MW}$ 、储能虚拟同步机 $10 \mathrm{MW}$. 随着清洁能源发电基地电力大规模外送和分布式清洁电 源渗透率不断增加，虚拟同步发电技术将面临新的挑战和 机遇.

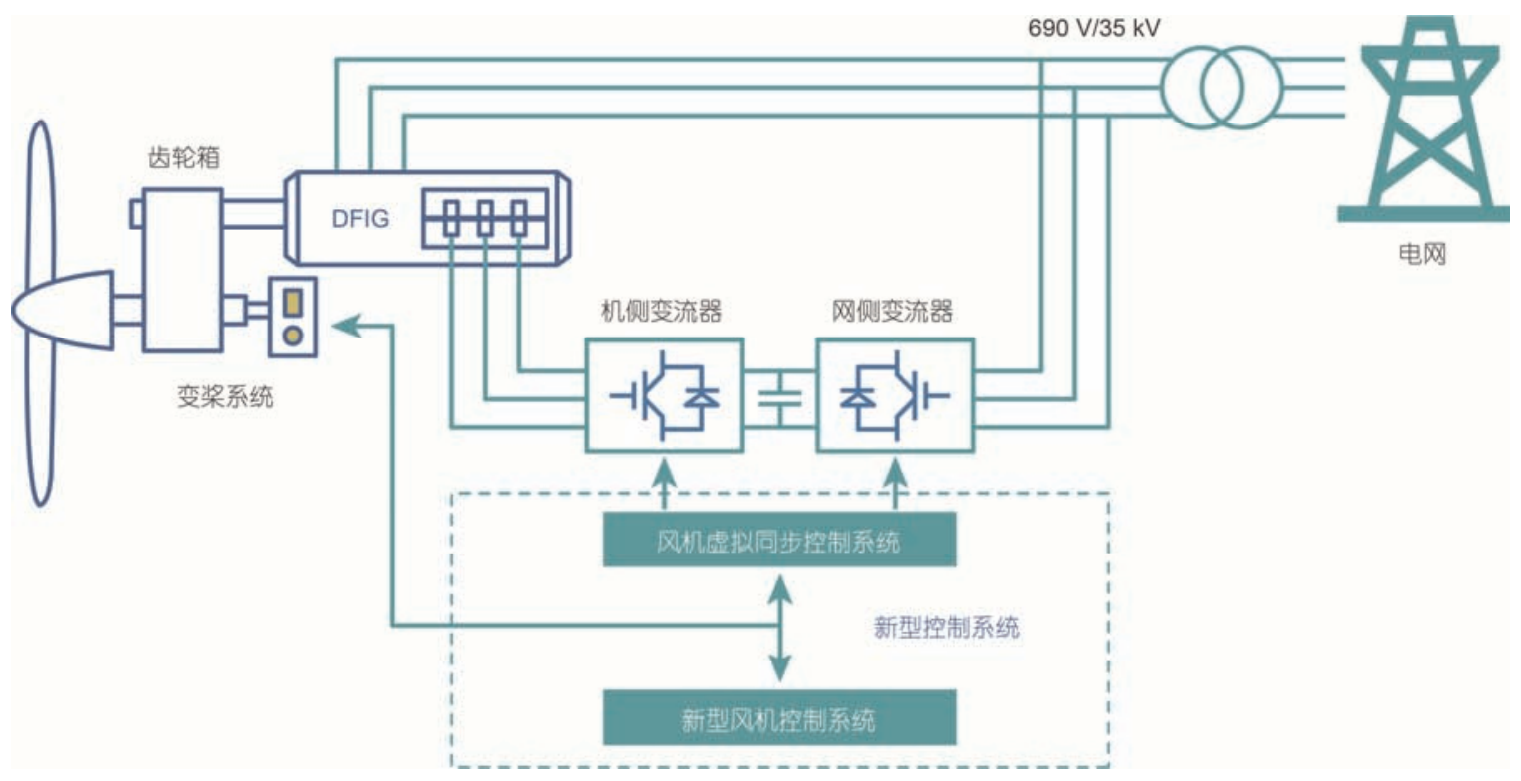

图 8 风机虚拟同步机工作示意图

Figure 8 Schematic diagram of virtual synchronous generators for wind power gengeration 


\section{2 特高压柔性直流输电技术}

随着大规模清洁能源的开发, 不同强度电网系统及无 源系统对于输电技术提出了更高要求. 特高压柔直输电技 术将在全球能源互联网中发挥重要作用.

柔性直流输电技术可实现对有功功率和无功功率在 潮流方向和幅值方面的解耦、快速、灵活控制, 同时对于 送端清洁能源以及受端弱交流系统、孤岛系统而言, 均可 实现较好的输送和运行效果, 克服了传统输电技术存在的 一些固有缺陷, 是构建全球特高压直流骨干网架的重要输 电技术. 目前柔性直流输电技术整体还处于 $500 \sim 800 \mathrm{kV}$ 、 3000 5000 MW 级水平, 在输送能力方面尚无法与传统直 流输电相媲美, 亟须发展更高电压等级、更大输送容量的 $800 \sim 1100 \mathrm{kV} 、 10 \sim 15 \mathrm{GW}$ 级特高压柔直输电技术. 为了构 建特高压柔性直流输电骨干网架, 需要进一步研制并完善 特高压柔性直流换流器、高压/特高压直流变压器(图9)、直 流电网潮流控制器(图 10)、高压/特高压直流断路器、特高 压直流电缆等核心设备.

\section{3 高温超导输电技术}

全球能源互联大电网中的重要能源节点、负荷节点以 及母线处存在大规模电力汇集和输送, 电力多向流动且频 繁换向, 母线节点上功率交换的量级巨大, 采用损耗低、容 量大的高温超导技术才能满足其功能需求. 一方面, 应用 超导技术建立超导化的超级母线, 可以更好适应接人资源 与负荷多样性, 在配备储能功能后, 更可以对量级巨大的 功率进行实时平衡, 有效平抑功率波动. 另一方面, 可基 于现有输电网络对部分线路进行超导化升级改造, 依托超 导输电线路占地面积小、质量轻、容量大等优点以取代 部分受空间或容量限制的常规线路, 是输电走廊受限情景 下的最佳技术路线. 图 11 所示为高温超导直流输电系统示 意图.

目前, 超导输电技术还受超导材料性能、成本以及低 温制冷技术等制约, 短期内难以大规模推广应用. 但从远 期来看, 随着超导带材临界温度的提高、生产加工成本的 降低、低温制冷效率以及可靠性的提升, 超导输电技术的 开发利用将可能极大地推动能源互联网建设.

\section{4 交直流混联大电网运行控制技术}

\subsection{1 智能调度技术}

全球互联电网是电力规模化集中汇集、远距离跨洲传 输、大范围灵活配置的重要基础平台, 具有接人电源类型 多元、设备类型多样、地域覆盖广泛等结构特征, 输送容 量大、潮流波动频繁、受扰行为复杂等运行特性, 对电网 运行全局态势感知能力、快速精确分析能力、新型电力设 备的灵活控制能力、大规模可再生能源接纳能力都提出了 更高要求和挑战. 因此, 需要采用智能调度技术以支持系 统运行.

\subsection{2 安全稳定控制技术}

随着电网跨国跨洲互联的推进, 电网动态特性的交互 影响日益显著, 区域电网之间故障传导范围明显增加, 高 比例新能源对电网运行控制的影响不断加剧, 且由于外部 环境多变并伴随极端灾害天气频发, 电网运行特性将更为 复杂. 因此, 需要加强未来电网安全稳定防御体系的架构 及构建.

3.4.3电网信息系统融合技术

随着电网自动化系统、大容量传输网、泛在传感网的 建设, 与互联网、能源网等深度融合从而构成电网信息物 理融合系统 (grid cyber physical system, GCPS). GCPS 通过 电网信息空间与物理空间的深度融合和实时交互来增加 或扩展新的功能, 安全、可靠、高效和实时监测或控制电 网物理设备或系统.

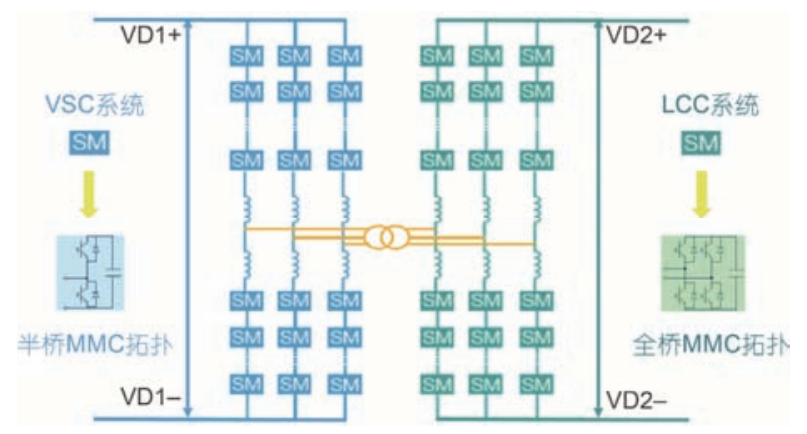

图 9 一种直流变压器示意图

Figure 9 Schematic diagram of a certain DC transformer

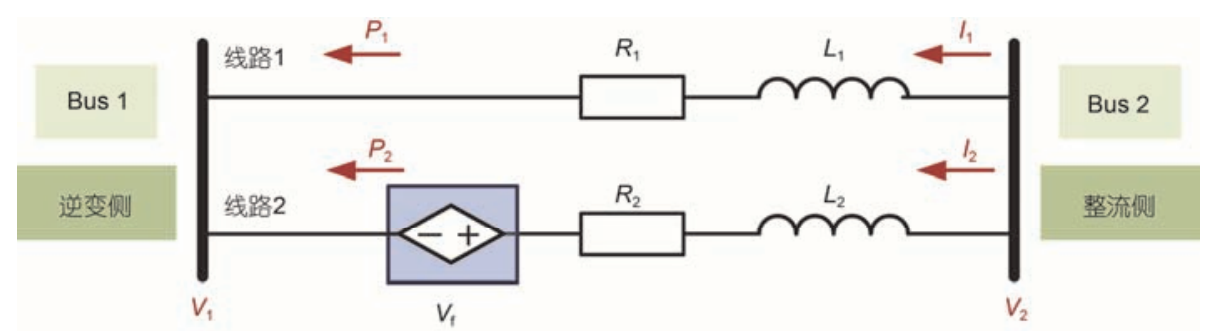

图 10 直流电网潮流控制器示意图

Figure 10 Schematic diagram of a power flow controller for DC grid 


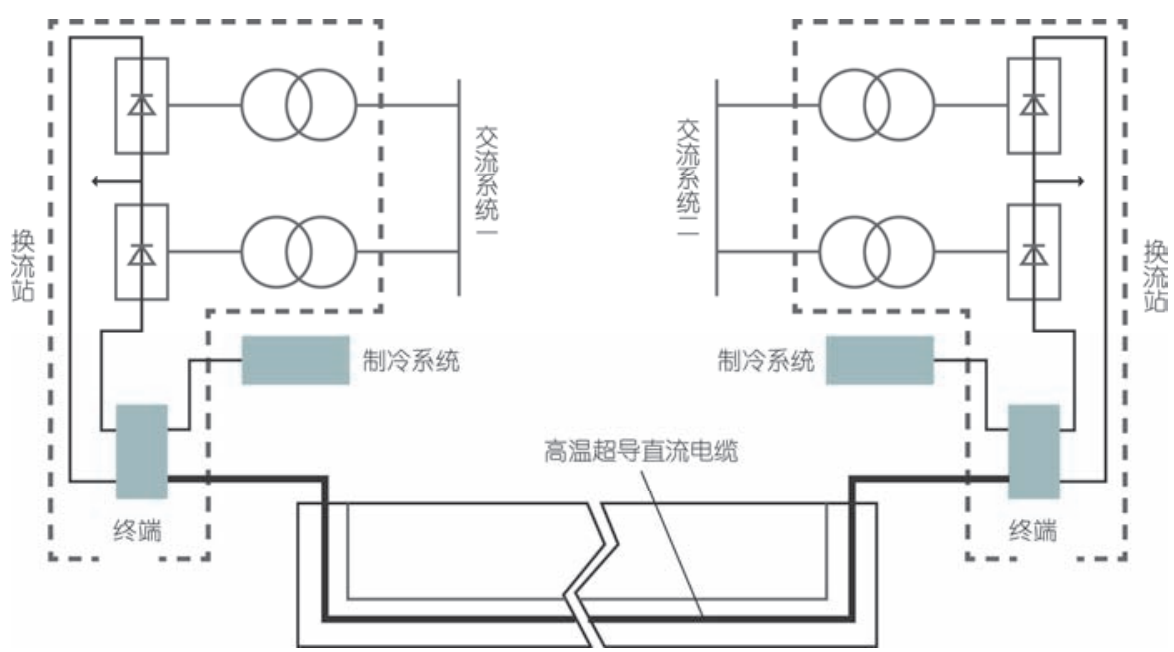

图 11 高温超导直流输电系统示意图

Figure 11 Schematic diagram of a superconducting HVDC transmission system

\subsection{4 洲际电力交易技术}

全球互联电网电力市场需要洲际市场、跨国市场、国 家市场等多级协调, 涵盖容量、电能、辅助服务以及输电 权等多种交易品种, 年度、日前、实时等多周期耦合电力 交易方式. 为了保障市场成员利益、维护电力市场规范运 营、规避市场风险、提升市场运行效率, 未来需要研究并 构建电力市场运营监管机制及体系, 研究洲际电力交易模 拟推演等技术.

\section{4 结论}

本文立足全球能源发展现状, 从应对能源挑战出发, 提出世界能源转型思路和具体方案. 根据全球经济社会、
能源电力发展趋势, 清洁能源资源禀赋、开发方式和电力 流格局研究成果, 在各洲能源互联网规划基础上, 提出了 全球能源互联网骨干网架方案和总体设想、建设规划, 并 对关键技术进行了分析和预测。总体上，全球能源互联网 在技术上具备可行性、经济上具备较高的综合价值和效益, 但仍需要在清洁能源开发技术、特高压柔直输电技术、高 温超导技术和大电网运行控制等方面实现进一步突破和完 善. 同时, 全球能源互联网的建设还需要全球各国在政治 理念上达成一致、相关政策上给予大力支持，营造良性的 市场环境，推动各行业技术全面进步，为促进清洁能源大 规模开发、全球配置和高效利用, 实现人类能源、经济、 社会、环境可持续发展提供解决方案.

\section{参考文献}

1 Jiang T, Li X C, Chao Q C, et al. Highlights and understanding of climate change 2014: Impacts, adaptation, and vulnerabilit (in Chinese). Clim Change Res, 2014, 10: 157-166 [姜粀, 李修仓, 巢清尘, 等. 《气候变化 2014: 影响、适应和脆弱性》的主要结论和新认知. 气 候变化研究进展, 2014, 10: 157-166]

2 Qin D H, Thomas S. Highlights of the IPCC working group I fifth assessment report (in Chinese). Clim Change Res, 2014, 10: 1-6 [秦大 河, Thomas S. IPCC 第五次评估报告第一工作组报告的亮点结论. 气候变化研究进展, 2014, 10: 1-6]

3 Zhang X H, Gao Y, Qi Y. Key Findings of the working group II contribution to the IPCC AR5 and their implications on the negotiations of the 2015 agreement (in Chinese). Clim Change Res, 2014, 10: 175-178 [张晓华，高云，祁悦. IPCC 第五次评估报告第二工作组报 告的主要结论对 2015 协议谈判的影响分析. 气候变化研究进展, 2014, 10: 175-178]

4 Zou J, Teng F, Fu S. The lateast progress in socioeconomic assessment of the mitigation of climate change-review of the IPCC fifth assessment working group III report (in Chinese). Clim Change Res, 2014, 10: 313-322 [邹瞕, 滕飞, 傅莎. 减缓气候变化社会经济评价 研究的最新进展一对 IPCC 第五次评估第三工作组报告的评述. 气候变化研究进展, 2014, 10: 313-322]

5 Liu Z Y. Global Energy Interconnection (in Chinese). Beijing: China Electric Power Press, 2015 [刘振亚. 全球能源互联网. 北京: 中 国电力出版社, 2015] 
6 Global Energy Interconnection Development Cooperation Organization. Global Energy Interconnection Backbone Network Frame Research (in Chinese). 2018 [全球能源互联网发展合作组织. 全球能源互联网骨干网架研究. 2018]

7 Global Energy Interconnection Development Cooperation Organization. Global Energy Interconnection Development Index (in Chinese). 2018 [全球能源互联网发展合作组织. 全球能源互联网发展指数. 2018]

8 Global Energy Interconnection Development Cooperation Organization. Chinese Energy Interconnection Development Research Report (in Chinese). 2017 [全球能源互联网发展合作组织. 中国能源互联网发展研究报告. 2017]

9 Global Energy Interconnection Development Cooperation Organization. Global Energy Interconnection Development and Outlook 2017 (in Chinese). 2017 [全球能源互联网发展合作组织. 全球能源互联网发展与展望 2017. 2017]

10 Global Energy Interconnection Development Cooperation Organization. Innovation Action Outline for Technical Equipment of Global Energy Interconnection 2018-2025 (in Chinese). 2018 [全球能源互联网发展合作组织. 全球能源互联网技术装备创新行动纲要 2018-2025. 2018] 
Summary for “全球能源互联网及关键技术”

\title{
Global Energy Interconnection vision and key technologies
}

\author{
Yuanbing Zhou \\ Global Energy Interconnection Development and cooperation Organization, Beijing 100031, China \\ E-mail: zhouyuanbing@geidco.org
}

Nowadays, climate change characterized by global warming exerts a huge impact on natural ecosystems and human society, threatening the sustainable development increasingly. Global warming is mainly caused by the accumulation of greenhouse gas emissions. Therefore, the realization of a low-carbon or even zero-carbon energy supply worldwide is the fundamental way to combat climate change.

Under such circumstances, this paper introduces the concept, vision, construction planning, and key technologies of Global Energy Interconnection. Actually, Global Energy Interconnection is a large-scale energy platform for the highly efficient deployment of clean energy around the world. Establishing Global Energy Interconnection has many advantages. On one hand, it can build a new pattern of energy development with breaking through the resources in time and space, and environment constraints, and achieve sufficient clean energy supply efficiently. On the other hand, it can stimulate economic growth while strengthening the momentum of development, releasing the innovation dividends, promoting cooperation, and accelerating the coordinating development of the world economy. In addition, it can create a beautiful new life for the global society while improving the natural environment, achieving the ecological sustainable development, promoting the harmonious development of mankind, and accelerating the upgrading of civilization. The main solutions and key technology foundations of establishing Global Energy Interconnection include the large-scale clean energy development technology, UHV transmission technology, and smart grid technology. Global Energy Interconnection is composed of intercontinental backbone grids, intracontinental backbone grids and different voltage-level grids within countries. The construction of Global Energy Interconnection is divided into three phases: 2035, 2050, and 2070. Eventually, the "9 Horizontal and 9 Vertical" global energy interconnection backbone grid will be formed, which will widely connect large-scale clean energy bases and load centers, promoting large-scale, wide-area and highly-efficient optimal global allocation of clean energy.

In general, the construction of Global Energy Interconnection has extremely high comprehensive benefits. Despite of technical feasibility, further improvements are still needed, including large-scale clean energy transmission and consumption, UHV flexible DC transmission technology, superconducting transmission technology, large power grid operation control technology and corresponding key equipments, etc. Moreover, the construction of Global Energy Interconnection also requires countries around the world to reach a consensus on political concepts and relevant policies, build a benign market environment, promote comprehensive technological progress in various industries, and provide solutions for large-scale development, global allocation, and efficient use of clean energy as well as the sustainable development of energy, economy, society and environment.

Global Energy Interconnection, backbone grid, clean energy development, UHV transmission technology, superconducting transmission technology, smart grid

doi: 10.1360/N972018-00757 